Experimental and theoretical determination of the plasmonic responses and shape distribution of colloidal metallic nanoparticles

A. Resano-Garcia, Y. Battie, A. En Naciri, S. Akil, and N. Chaoui

Citation: The Journal of Chemical Physics 142, 134108 (2015); doi: 10.1063/1.4916917

View online: http://dx.doi.org/10.1063/1.4916917

View Table of Contents: http://aip.scitation.org/toc/jcp/142/13

Published by the American Institute of Physics

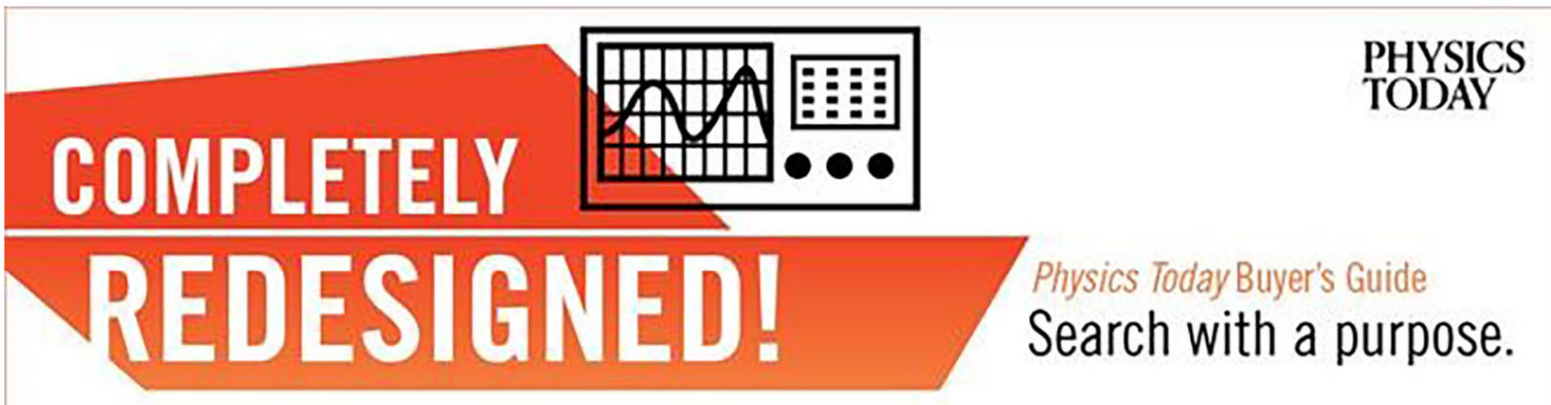




\title{
Experimental and theoretical determination of the plasmonic responses and shape distribution of colloidal metallic nanoparticles
}

\author{
A. Resano-Garcia, Y. Battie, ${ }^{\text {a) }}$ A. En Naciri, S. Akil, and N. Chaoui \\ LCP-A2MC, Institut Jean Barriol, Université de Lorraine, 1 Bd Arago, 57070 Metz, France
}

(Received 6 November 2014; accepted 14 March 2015; published online 6 April 2015)

\begin{abstract}
The optical properties of gold and silver nanoparticles (NPs) dispersed in water and distributed in shape are investigated by introducing a shape distributed effective medium theory (SDEMT). This model takes into account the variation of depolarization parameter induced by a NP shape distribution. Simulations show that the shape distribution induces an inhomogeneous broadening and a decrease of the amplitude of the plasmon band. The number of plasmon bands and their positions depend on both the mean value of depolarization parameter and the NP material. By fitting the measured absorption spectra with the SDEMT, we unambiguously demonstrate that the depolarization parameter distribution, i.e., the shape distribution of nanoparticles can be deduced from absorption spectra. (C) 2015 AIP Publishing LLC. [http://dx.doi.org/10.1063/1.4916917]
\end{abstract}

\section{INTRODUCTION}

Noble metal nanoparticle (NP) exhibits a very intense color which is absent in the bulk material. This property is attributed to the surface plasmon resonance (SPR) induced by the collective oscillation of their free conduction electrons. The characteristic of the SPR band depends on the NP size, shape, and environment. ${ }^{1-4}$ This tunability makes noble metallic NP an ideal candidate for a wide range of applications such as chemical sensor, ${ }^{5}$ white light processing, ${ }^{6}$ and solar cells. ${ }^{7}$

The design of NP devices and their optical characterization require the development of models which describes the optical properties of NPs. The extinction cross section of isolated spherical NPs is well described by the Mie theory. ${ }^{1,8}$ Amendola and Meneghetti ${ }^{9}$ and Garrelie et al. ${ }^{10}$ have introduced the size distribution in the Mie theory by weighting the extinction cross section of individual NPs by the size distribution. However, the classical Mie theory does not consider interactions between NPs and shape distribution. In other words, this approach is only restricted to highly diluted spherical NPs. Eustis and ElSayed ${ }^{11}$ have proposed to determine the aspect ratio distribution of gold nanorods by fitting their absorption spectra band with the Gans theory. The contribution of each nanorod is taken into account by summing their absorption. However, the authors focus their investigation on the longitudinal SPR (L-SPR) band and neglect the influence of the shape distribution on the transversal plasmon band (T-SPR) and the interband transition of NPs. Moreover, the complex effective dielectric function of composite medium, which is a key parameter for ellipsometric investigation and designing optical devices, cannot be directly extracted from the Mie theory or Gans theory. Effective medium theories, which approximate the composite material as a homogeneous medium, allow calculating the complex dielectric function and require few computing resources. However, this homogenization procedure can

\footnotetext{
a) Author to whom correspondence should be addressed. Electronic mail: yann.battie@univ-lorraine.fr
}

introduce some errors, and the quality of the approximation must be evaluated by far-field measurements. This usual approach can be considered since the scattering cross section of the metallic NPs is negligible, i.e., for NP size smaller than the wavelength. The Maxwell Garnett theory, ${ }^{12}$ which is based on the Lorentz local field, is often used to predict the SPR band of monodispersed spherical NPs. ${ }^{13-16}$ This model was recently extended to describe the optical properties of spherical NPs distributed in size by averaging their polarizability. ${ }^{17,18}$ However, preparation routes unavoidably conduct to NPs showing a shape distribution which induces drastic changes in the optical properties of NP assembly. ${ }^{19-22}$ Indeed, by combining Gibbs free energy calculation to discrete dipole approximation, Noguez et al. ${ }^{23-25}$ have shown that the morphology and so the optical properties of NPs depend on the temperature used to synthesize them. In addition, as reported by Sayah et al.,$^{19}$ silver prolates grown in a mesoporous silica matrix exhibit a large length distribution which induces an inhomogeneous broadening of the L-SPR bands. This inhomogeneous broadening is not taken into account in classical effective medium theory.

Several attempts have been made to introduce the NP shape distribution in effective medium theory. Toudert et al. ${ }^{21}$ have introduced the NP shape distribution in the Yamaguchi model. ${ }^{26}$ However, this model can only be applied for two dimensional arrays of NPs. Moreover, it requires a preliminary estimation of the pair correlation function of NPs by transmission electron microscopy (TEM). Another two dimensional effective medium theory was recently introduced to analyse ellipsometric measurements performed on gold nanoisland films. ${ }^{20}$ This theory takes into account both the shape distribution of oblate nanoislands and the anisotropy induced by their two dimensional orientation. However, this model neglects charge image effects. Based on the mean field approximation, Bohren and Huffman ${ }^{8}$ have proposed a shape distributed effective medium theory (SDEMT) which extends the Maxwell Garnett theory by considering spheroidal NPs distributed in shape. By applying SDEMT in a low NP volume 
fraction limit, Goncharenko et al. ${ }^{27}$ have derived a spectral Bergman representation of the effective dielectric function. This approach was recently used to design broadband epsilonnear-zero composites. ${ }^{28}$ Moreover, a same approach was used to extend the Bruggeman theory in order to describe the percolation threshold of intermixed components distributed in shape. ${ }^{29,30}$ However, these authors consider unphysical distributions such as a uniform steplike shape distribution. ${ }^{27}$ Moreover, no direct comparison with experimental results has been proposed to validate this approach.

In this paper, the influence of NP shape distribution on the optical properties of colloidal solution of gold and silver NPs is highlighted through several simulations based on SDEMT. By considering a Gaussian distribution, we demonstrate that the SPR energies mainly depend on the mean value of the depolarization parameter while the width of the SPR bands is directly correlated to the standard deviation of the NP shape distribution. By fitting absorption measurements with SDEMT, we unambiguously demonstrate that the distribution of depolarization parameter of colloidal gold and silver solutions, which is the optical signature of the shape distribution, can be extracted from their absorption spectra.

\section{THEORY}

\section{A. SDEMT}

The effective dielectric function $\varepsilon_{\text {eff }}$ of a composite material, which consists in a collection of randomly oriented spheroidal NPs embedded in a matrix, is related to the spatial averages of the displacement $\langle\mathbf{D}\rangle$ and electric field $\langle\mathbf{E}\rangle$,

$$
\langle\mathbf{D}\rangle=\varepsilon_{e f f}\langle\mathbf{E}\rangle .
$$

By considering the mean field approximation, the averages of the displacement and electric field can be decomposed into two contributions, ${ }^{8}$

$$
\begin{gathered}
\langle\mathbf{D}\rangle=(1-f) \varepsilon_{m}\left\langle\mathbf{E}_{\mathbf{m}}\right\rangle+f \varepsilon_{n p}\left\langle\mathbf{E}_{\mathbf{n p}}\right\rangle, \\
\langle\mathbf{E}\rangle=(1-f)\left\langle\mathbf{E}_{\mathbf{m}}\right\rangle+f\left\langle\mathbf{E}_{\mathbf{n p}}\right\rangle,
\end{gathered}
$$

where $f$ is the volume fraction occupied by the NPs. The subscripts $m$ and $n p$ refer to the matrix and the NPs, respectively. $\varepsilon_{m}$ and $\varepsilon_{n p}$ are the dielectric function of the surrounding medium and NPs, respectively. By considering the quasistatic limit, i.e., for NP size smaller than the wavelength, the electric field inside NPs is proportional to electric field in the matrix,

$$
\mathbf{E}_{\mathbf{n p}}=\lambda \mathbf{E}_{\mathbf{m}} .
$$

For randomly oriented spheroidal NPs, the parameter $\lambda$ is given by $^{8}$

$$
\lambda\left(L_{1}, L_{2}\right)=\frac{1}{3} \sum_{i=1}^{3} \frac{\varepsilon_{m}}{\varepsilon_{m}+L_{i}\left(\varepsilon_{N P}-\varepsilon_{m}\right)},
$$

where $\mathrm{L}_{1}, \mathrm{~L}_{2}$, and $\mathrm{L}_{3}$ are the depolarization parameters of spheroidal NPs along their three principal axes. These parameters, which vary from 0 to 1 , only depend on the NP shape. Moreover, they must respect the following sum rule:

$$
L_{1}+L_{2}+L_{3}=1 \text {. }
$$

The average electric field inside NPs is calculated by weighting the function $\lambda\left(L_{1}, L_{2}\right)$ of individual NPs by the distribution of depolarization parameters $\mathrm{P}\left(L_{1}, L_{2}\right)$,

$$
\left\langle\mathbf{E}_{\mathbf{n p}}\right\rangle=\iint \mathrm{P}\left(L_{1}, L_{2}\right) \lambda\left(L_{1}, L_{2}\right) d L_{1} d L_{2}\left\langle\mathbf{E}_{\mathbf{m}}\right\rangle .
$$

Equation (7) assumes that the field inside the matrix is homogeneous. In other words, the volume fraction must be sufficiently small $(f<30 \%)^{1}$ to avoid hot spots induced by the interaction between NPs.

Combining Eqs. (1)-(3) and (7), the effective dielectric function of a medium which consists in a collection of spheroids distributed in shape and randomly oriented can be expressed by the following equation: ${ }^{8}$

$$
\varepsilon_{e f f}=\frac{(1-f) \varepsilon_{m}+f \varepsilon_{n p} \iint \mathrm{P}\left(L_{1}, L_{2}\right) \lambda\left(L_{1}, L_{2}\right) d L_{1} d L_{2}}{(1-f)+f \iint \mathrm{P}\left(L_{1}, L_{2}\right) \lambda\left(L_{1}, L_{2}\right) d L_{1} d L_{2}} .
$$

The effective dielectric function $\varepsilon_{\text {eff }}$ given by Eq. (8) allows describing the optical properties of a large number of NP shapes such as oblate, prolate, or nanospheres. For monodispersed spherical NPs, this equation leads to the well known Maxwell Garnett theory. ${ }^{12}$ In other words, Eq. (8) is a general formulation of classical effective medium theories. The effective absorption coefficient of a composite medium which contains a volume fraction spheroidal NPs distributed in shape can be evaluated from

$$
\alpha=\frac{4 \pi}{\lambda_{0}} \operatorname{Im}\left(\sqrt{\varepsilon_{e f f}}\right),
$$

where $\lambda_{0}$ is the wavelength of light in vacuum.

\section{B. Radius distributed Mie theory}

By solving the Maxwell equation in a spherical frame, the Mie theory ${ }^{1,8}$ allows calculating the extinction cross section of a spherical NP embedded in a matrix,

$$
\sigma_{e x t}(R)=\frac{\lambda_{0}^{2}}{2 \pi \varepsilon_{m}} \sum_{n=1}^{+\infty}(2 n+1) \operatorname{Re}\left\{a_{n}(R)+b_{n}(R)\right\},
$$


with

$$
\begin{aligned}
& a_{n}(R)=\frac{\sqrt{\varepsilon_{n p}} \Psi_{n}\left(k_{n p} R\right) \Psi_{n}^{\prime}\left(k_{m} R\right)-\sqrt{\varepsilon_{m}} \Psi_{n}\left(k_{m} R\right) \Psi_{n}^{\prime}\left(k_{n p} R\right)}{\sqrt{\varepsilon_{n p}} \Psi_{n}\left(k_{n p} R\right) \xi_{n}^{\prime}\left(k_{m} R\right)-\sqrt{\varepsilon_{m}} \Psi_{n}\left(k_{m} R\right) \xi_{n}^{\prime}\left(k_{n p} R\right)}, \\
& b_{n}(R)=\frac{\sqrt{\varepsilon_{m}} \Psi_{n}\left(k_{n p} R\right) \Psi_{n}^{\prime}\left(k_{m} R\right)-\sqrt{\varepsilon_{n p}} \Psi_{n}\left(k_{m} R\right) \Psi_{n}^{\prime}\left(k_{n p} R\right)}{\sqrt{\varepsilon_{n p}} \Psi_{n}\left(k_{n p} R\right) \xi_{n}^{\prime}\left(k_{m} R\right)-\sqrt{\varepsilon_{n p}} \Psi_{n}\left(k_{m} R\right) \xi_{n}^{\prime}\left(k_{n p} R\right)},
\end{aligned}
$$

where $k_{n p}$ and $k_{m}$ are the wave number inside the NP and the matrix, respectively. $\mathrm{R}$ is assimilated to the NP radius, while $\lambda_{0}$ is the wavelength in vacuum. $\Psi_{\mathrm{n}}$ and $\xi_{\mathrm{n}}$ are the n order RiccattiBessel functions, while $a_{n}$ and $b_{n}$ are the Mie coefficients. The summation index $\mathrm{n}$ gives the order of spherical multipole excitation. By considering sufficiently diluted spherical NPs, the interaction between metallic NPs can be neglected. Thus, the effective absorption coefficient of a composite medium which contains a volume fraction $f$ of spherical NPs distributed in size can be evaluated from

$$
\alpha=\frac{3 f}{4 \pi \bar{R}^{3}} \int P_{R}(R) \sigma_{e x t}(R) d R,
$$

where $P_{R}(R)$ is the NP radius distribution.

\section{SIMULATIONS}

The effective complex dielectric function and absorption coefficient of gold and silver spheroidal NPs embedded in host liquid matrix are simulated from Eqs. (8) and (9) for several NP shape distributions. By considering that the NP size is higher than $5 \mathrm{~nm},{ }^{17}$ the intrinsic confinement induced by the limitation of the electron mean free path can be neglected. ${ }^{1}$ In other words, $\varepsilon_{\mathrm{np}}$ is assimilated to the bulk dielectric function of silver ${ }^{31}$ and gold. ${ }^{32}$ Water is used as host matrix. The NP volume fraction is fixed to $1 \%$. A normalized Gaussian distribution of depolarization parameter is considered,

$$
\mathrm{P}\left(L_{1}, L_{2}\right)=C e^{-\frac{\left(L_{1}-\overline{L_{1}}\right)^{2}}{2 \sigma_{1}^{2}}-\frac{\left(L_{2}-\overline{L_{2}}\right)^{2}}{2 \sigma_{2}^{2}}-\frac{\left(L_{3}-\overline{L_{3}}\right)^{2}}{2 \sigma_{3}^{2}}},
$$

where $\sigma_{i}$ and $\overline{L_{i}}$ are the standard deviation and mean value of $L_{i}$, respectively ( $\left.\mathrm{i}=1,2,3\right)$. The mean values of depolarization parameters must respect Eq. (6). We apply the convention introduced by Bohren and Huffman, ${ }^{8} \overline{L_{1}} \leq \overline{L_{2}} \leq \overline{L_{3}}$. The constant $C$ is used to normalize the distribution in the two dimensional space $\left(L_{1}, L_{2}\right)$ of depolarization parameter. Despite no analytical solution of Eq. (8) can be found for this distribution, this distribution is more realistic than the ones previously used in Ref. 27.

First, the simulations of the optical response of aqueous colloidal solutions of Au and Ag NPs are performed using SDEMT without considering the shape dispersion (Fig. 1). In other words, the standard deviations of the depolarization parameters are equal to zero. Four NP shapes are investigated: spherical NPs $\left(\overline{L_{1}}=\overline{L_{2}}=\overline{L_{3}}=0.33\right)$, prolates $\left(\overline{L_{1}}\right.$ $\left.=0.1, \overline{L_{2}}=\overline{L_{3}}=0.45\right)$, oblates $\left(\overline{L_{1}}=\overline{L_{2}}=0.2, \overline{L_{3}}=0.6\right)$, and non-degenerated arbitrary spheroids $\left(\overline{L_{1}}=0.1, \overline{L_{2}}=0.2\right.$, $\left.\overline{L_{3}}=0.7\right)$. The imaginary part of the effective dielectric function $\left(\varepsilon_{\text {effi }}\right)$ of spherical $\mathrm{Ag}$ and Au NPs exhibits a narrow SPR band centred at $3.2 \mathrm{eV}$ and $2.3 \mathrm{eV}$, respectively. Note that the shoulder located at $3.4 \mathrm{eV}$ on the spectra of Ag NPs is an artefact which comes from the Palik dielectric function of Ag. ${ }^{31}$ Indeed, the tabulated dielectric function of $\mathrm{Ag}$ is made by juxtaposing, at $3.4 \mathrm{eV}$, the Winsemius et al. ${ }^{33}$ and the Leveque et al. ${ }^{34}$ databases. In other words, this shoulder is only due to a poor continuity of bulk Ag dielectric function database.
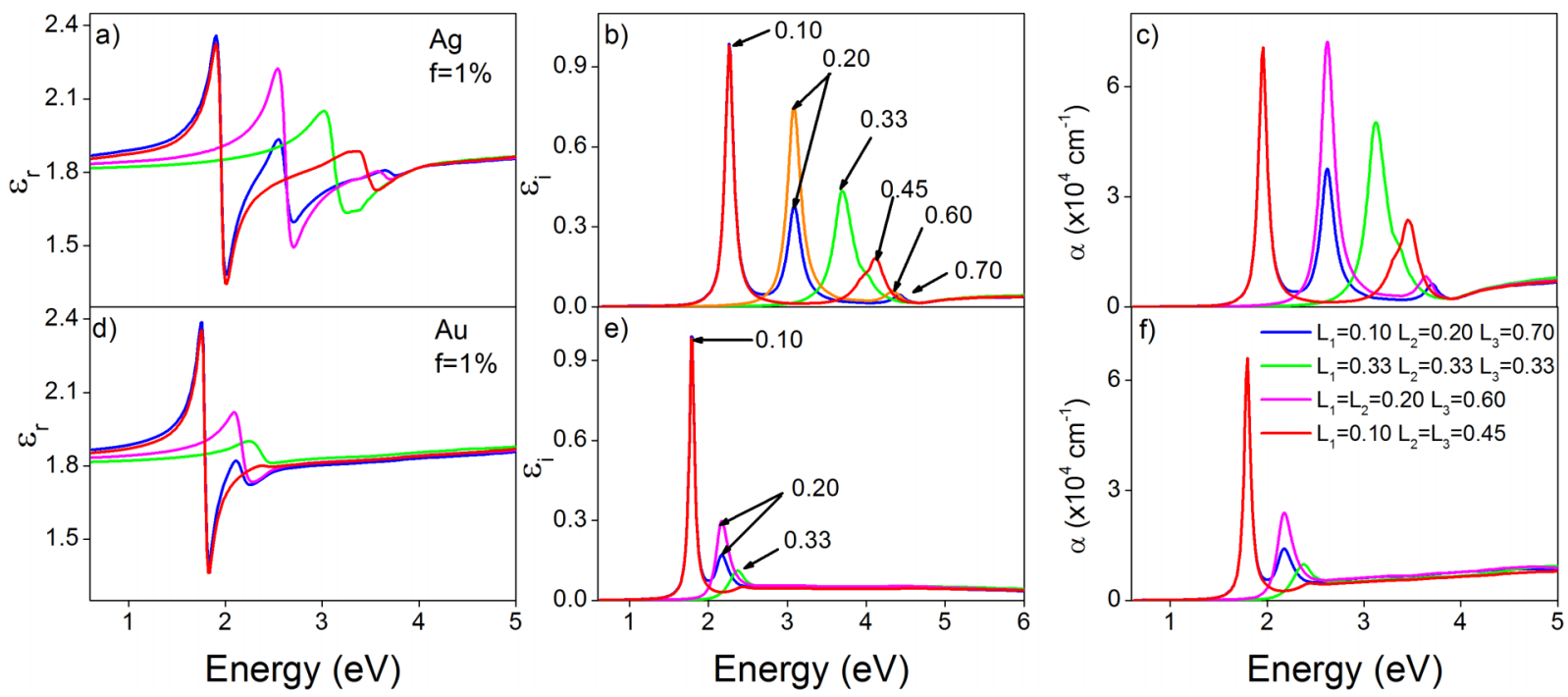

FIG. 1. ((a), (b), (d), and (e)) Complex effective dielectric function and ((c) and (f)) effective absorption spectra of ((a)-(c)) Ag and ((d)-(f)) Au monodisperse ellipsoids in water. The NP volume fraction is $1 \%$. 

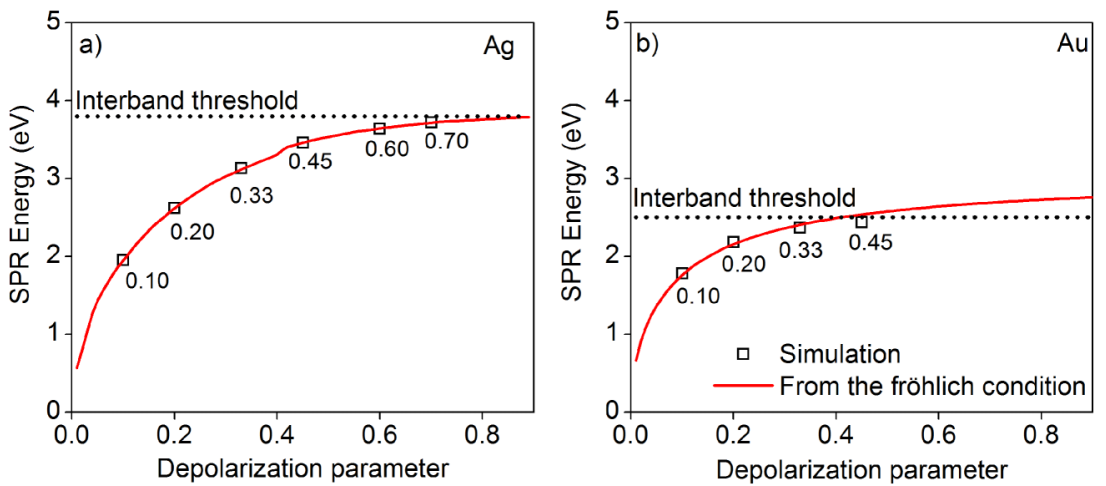

FIG. 2. Influence of the depolarization parameter on the SPR energy of (a) $\mathrm{Ag}$ and (b) Au NPs in water. The red curve is obtained from Fröhlich condition (15), while the empty square is deduced from the simulated spectra of monodisperse ellipsoidal NPs by considering a $1 \%$ volume fraction.

The SPR energy depends on the depolarization parameter $\left(\mathrm{L}_{\mathrm{i}}\right)$. As shown in Figure 2, in the low concentration limit, the SPR energy $\left(\omega_{\mathrm{ri}}\right)$ is given by the Fröhlich condition ${ }^{1}$ which occurs near the virtual pole of Eq. (8),

$$
0=\varepsilon_{n p r}\left(\omega_{r i}\right)+\frac{1-L_{i}}{L_{i}} \varepsilon_{m r}\left(\omega_{r i}\right),
$$

where $\varepsilon_{\mathrm{npr}}$ and $\varepsilon_{\mathrm{mr}}$ are the real parts of the dielectric functions of the NP and the matrix, respectively.

In accordance with the Kramers-Kronig relations, a large variation of the real part of the effective dielectric function occurs close to the SPR (Fig. 1). The interband transition threshold, located at $2.5 \mathrm{eV}$ and $3.8 \mathrm{eV}$ for $\mathrm{Au}$ and $\mathrm{Ag}$, respectively, is clearly observed (Fig. 1). For energy higher than this threshold, $\varepsilon_{\text {effi }}$ is dominated by the interband contribution and becomes roughly constant. Due to the lifting of degeneracy, the SPR band of oblate and prolate Ag NPs is split into two SPR modes. The T-SPR mode, located at energy higher than the SPR energy of spherical NPs, is excited by a light polarized along the minor axes of the NPs. ${ }^{2}$ The L-SPR mode, observed at a lower energy than the SPR mode of spherical NPs, is selectively excited with a polarization parallel to the major axes
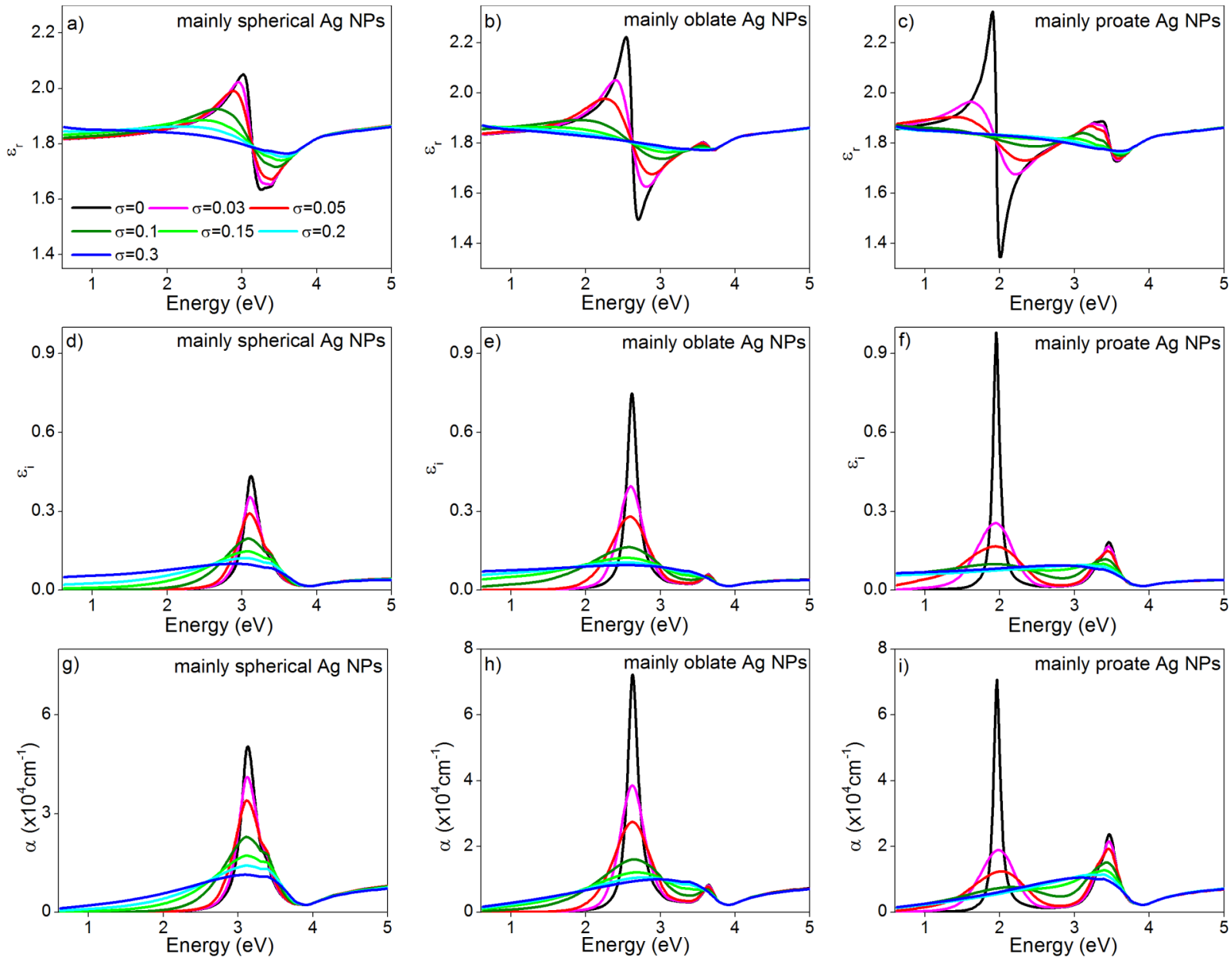

FIG. 3. Influence of the standard deviation $\sigma$ of depolarization parameters on ((g)-(i)) the effective absorption coefficient and ((a)-(c)) the real part and ((d)-(f)) imaginary part of the effective dielectric function of silver NPs in water which are mainly composed of ((a), (b), and (g)) spherical, ((b), (e), and (h)) oblate, and ((c), (f), and (i)) prolate NPs. The NP volume fraction is $1 \%$. 
of the NPs. ${ }^{2}$ The amplitude of the T-SPR band is smaller than the L-SPR one. Indeed, the T-SPR band is close to the interband threshold. In this spectral range, the imaginary part of the NP dielectric function and so the optical losses increase. In other words, the T-SPR mode is damped by the interband transitions. Moreover, the excitation along the NP minor axis is strongly reduced by depolarization effects. ${ }^{1,8,19}$ This depolarization effect comes from the presence of microscopic electric fields due to the induced polarization charges after the application of the external field. Due to the higher contribution of the $\mathrm{Au}$ interband transitions, the T-SPR band of the considered prolate and oblate Au NPs is not observed (Fig. 1). In other words, the spectra of prolate or oblate Au NPs are mainly dominated by the L-SPR band. As arbitrary Ag spheroids are completely non-degenerated, three SPR bands are observed in their spectra. However, due to the presence of interband transitions, the spectrum of Au arbitrary spheroids exhibits only two SPR bands. In agreement with Eq. (15) and Figure 2, these bands are assigned to $\overline{L_{1}}=0.1$ and $\overline{L_{2}}=0.2$.

Figures 3 and 4 illustrate the effect of the shape distribution on the optical properties of a collection of silver and gold NPs which mainly contains spherical, oblate, or prolate NPs. Simulations are performed by changing the standard deviation of depolarization parameter assuming that $\sigma_{1}=\sigma_{2}=\sigma_{3}$.
Similar results are obtained for spherical, prolate, and oblate NPs. Indeed, the SPR energy is slightly influenced by standard deviation. In accordance with Eq. (15), the SPR energy is mainly related to the mean value of depolarization parameter. Indeed, the considered Gaussian distribution is symmetric about its mean value. Thus, the shift introduced by NPs which have a larger depolarization parameter than the mean value is compensated by the one induced by NPs which have a smaller depolarization parameter. According to Eq. (15), the SPR energies can be used to estimate the mean value of depolarization parameters and so the overall NP shape. As each NP has specific depolarization parameters, the shape distribution induces an inhomogeneous broadening ${ }^{19}$ and a large decrease of the amplitude of the SPR bands. These effects are more pronounced for the L-SPR of prolate and oblate NPs. This mode corresponds to a small value of depolarization parameter. Indeed, for a low mean value of depolarization parameter, a small variation of depolarization parameter induces a large shift of the plasmon band (Fig. 2). This sensitivity drastically decreases as the mean value of depolarization parameter increases. This behavior is in agreement with recent experimental results which show that the L-SPR band of silver prolate distributed in length is more broadened than the T-SPR band. ${ }^{19}$ For high standard deviation, the T-SPR and L-SPR bands of
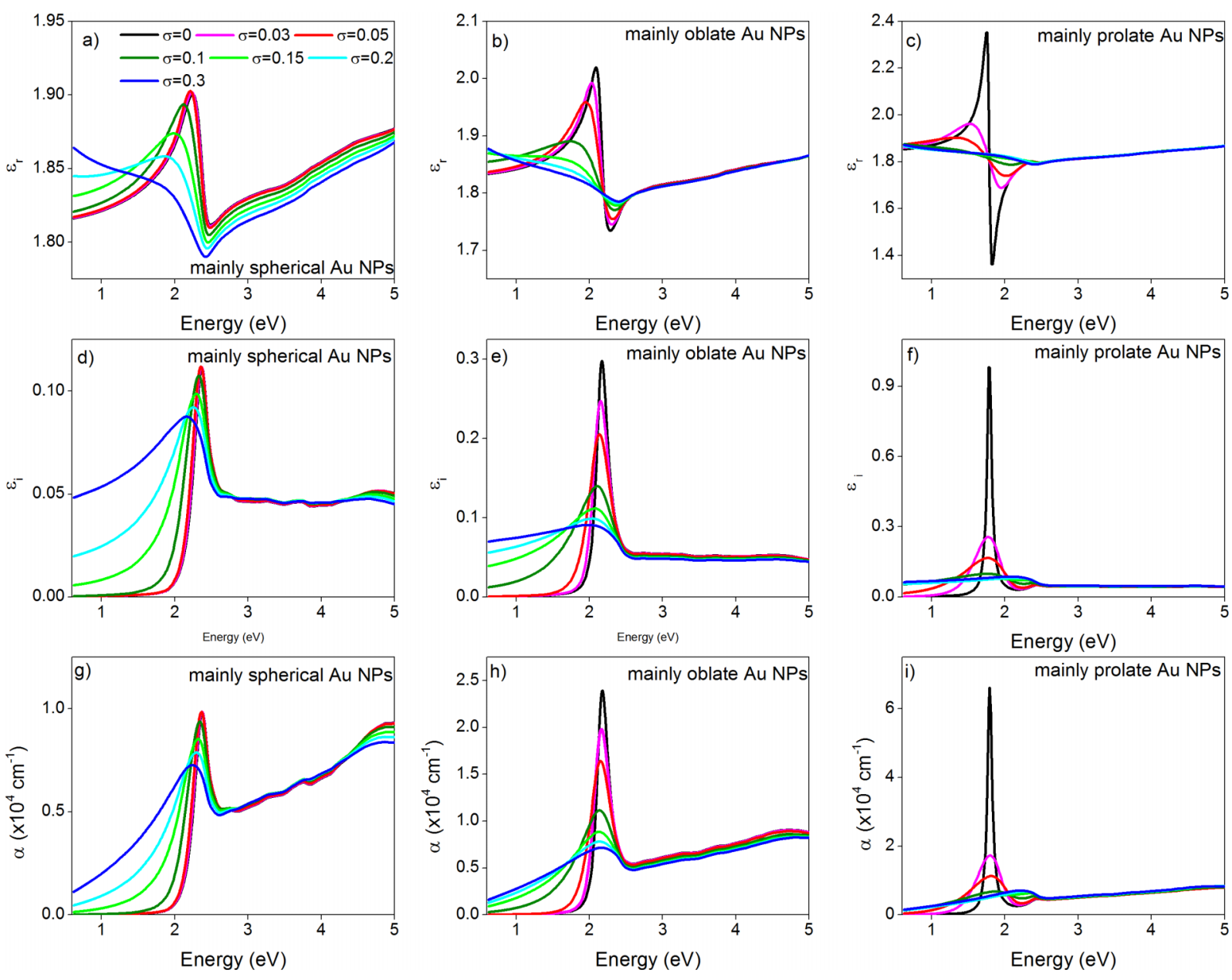

FIG. 4. Influence of the standard deviation $\sigma$ of depolarization parameters on ((g)-(i)) the effective absorption coefficient and ((a)-(c)) the real part and ((d)-(f)) imaginary part of the effective dielectric function of gold NPs in water which are mainly composed of ((a), (b), and (g)) spherical, ((b), (e), and (h)) oblate, and ((c), (f), and (i)) prolate NPs. The NP volume fraction is $1 \%$. 
oblate and prolate NPs overlap and only one broad SPR band is observed. This SPR band loses its symmetry and acquires a pronounced absorption tail. In other words, the SPR band asymmetry can be used to evaluate qualitatively the width of the NP shape dispersion.

In order to give a quantitative analysis, the effective absorption coefficient of NPs at $4.5 \mathrm{eV}\left(\alpha_{4.5 \mathrm{eV}}\right)$ and $2 \mathrm{eV}\left(\alpha_{2} \mathrm{eV}\right)$ is calculated as a function of the volume fractions. By randomly selecting the mean value of depolarization and the standard deviations of the distribution, $100 \mathrm{NP}$ shape distributions are generated. The standard deviation of the distribution varies in the $0-0.3$ range. As shown in Figure 5, $\alpha_{2} \mathrm{eV}$ exhibits a strong dependence on both the volume fraction and the distribution of depolarization parameter. Indeed, in the visible spectral range, the optical properties of $\mathrm{Ag}$ and $\mathrm{Au}$ NPs are related to their plasmon bands. As the SPR band energy, amplitude, and width are mainly defined to the NP shape distribution (Figs. 3 and 4), the $\alpha_{2} \mathrm{eV}$ values are highly dispersed. On the contrary, a smaller dependence between the shape distribution and the effective absorption coefficient is observed at $4.5 \mathrm{eV}$. $\alpha_{4.5 \mathrm{eV}}$ linearly increases with the NPs' volume fraction. Due to its predominant interband transition, the effective absorption coefficient of Au NPs at $4.5 \mathrm{eV}$ is higher than the Ag NPs' one. The influence of the shape distribution on the $\alpha_{4.5 \mathrm{eV}}$ increases as the volume fraction increases. However, by considering the extreme value of $\alpha_{4.5 \mathrm{ev}}$, the error induced by the NP shape distribution on the estimation of the volume fraction from $\alpha_{4.5 \mathrm{eV}}$ is less than $7 \%$. In other words, $\alpha_{4.5 \mathrm{eV}}$ can be directly used to extract the concentration of $\mathrm{Au}$ and $\mathrm{Ag}$ NPs.

\section{EXPERIMENTS: APPLICATION TO COLLOIDAL SOLUTIONS}

Three colloidal solutions of gold NPs (Au1, Au2, and Au3) and one colloidal solution of silver NPs (Ag1) dispersed in water are investigated. Au1 and Ag1 are purchased from Sigma Aldrich and are used as received. The fabrication of
$\mathrm{Au} 2$ was performed according to El-Sayed and Nikoobakht ${ }^{35}$ using the so-called seed-mediated growth method. In such synthesis, a seed solution is added to a growth one containing additional metal salt and structure-direction agent to obtain high aspect ratio. Here, silver ion concentration and seed $/ \mathrm{Au}^{3+}$ were varied to achieve fine tuning of the nanorod dimensions. This results in changes of the absorption wavelength of gold NPs. Au3 solution is synthesized by a home built laser ablation setup. ${ }^{36} \mathrm{Au} 3$ solution was prepared by laser ablation in liquid. Briefly, the laser ablation of a pure $(99.99 \%)$ gold target was conducted in water using a frequency doubled (532 nm, 6-ns pulse duration) Nd-YAG laser (Continuum Surelite I). The laser fluence and pulses repetition rate were $2.3 \mathrm{~J} \mathrm{~cm}^{-2}$ and $10 \mathrm{~Hz}$, respectively. TEM was performed with a Phillips CM200 microscope operating at $200 \mathrm{kV}$. The TEM grids were prepared by evaporating a drop of colloidal NP solution on a copper TEM grid. The absorption spectra were recorded in the UV and visible spectral ranges with a Horiba Jobin-Yvon spectrometer.

Figures 6(a) and 6(d) show the TEM images of Au1 and Ag1. All NPs have an almost spherical shape. The apparent NPs' radius distribution follows a lognormal distribution. The Au1 particles are faceted than Ag1. The mean radius of Ag1 and Au1 is $4.9 \mathrm{~nm}$ and $15.5 \mathrm{~nm}$, respectively. The absorption spectra of Au1 and Ag1 (Figs. 6(b) and 6(e)) exhibit a SPR band located at $3.13 \mathrm{eV}$ and $2.36 \mathrm{eV}$, respectively. TEM images give a two dimensional projection of NPs. To confirm the nearly spherical shape of NPs, the measured absorption spectra are compared to the fitted ones from the radius distributed Mie theory. The NP size distributions of Au1 and Ag1 (Figs. 6(a) and 6(d)) are set to the ones obtained from a statistical analysis of TEM images performed over 250 NPs. Note that the volume fraction of NPs is the single free parameter. A great agreement was found between the experimental spectra of $\mathrm{Ag} 1$ and $\mathrm{Au} 1$ and the calculated one from the radius distributed Mie theory, confirming that Ag1 and Au1 NPs have a nearly spherical shape. The volume fractions of Ag1 and Au1 are
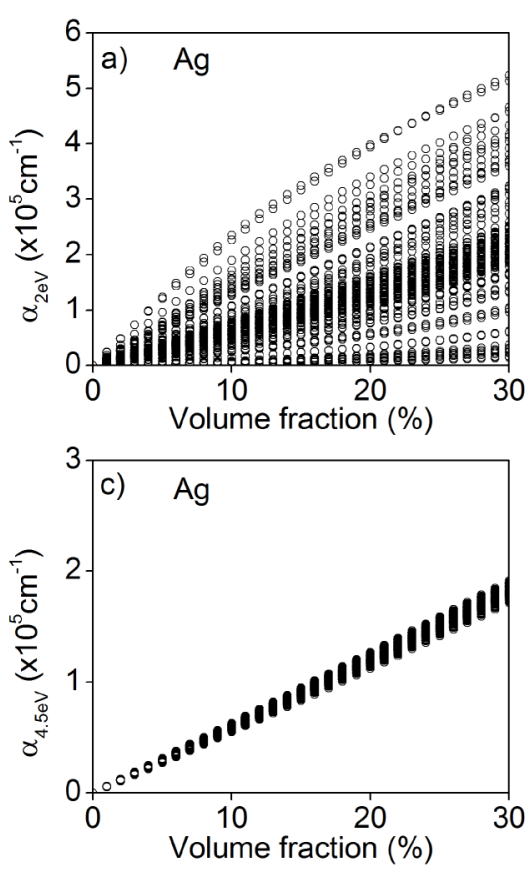
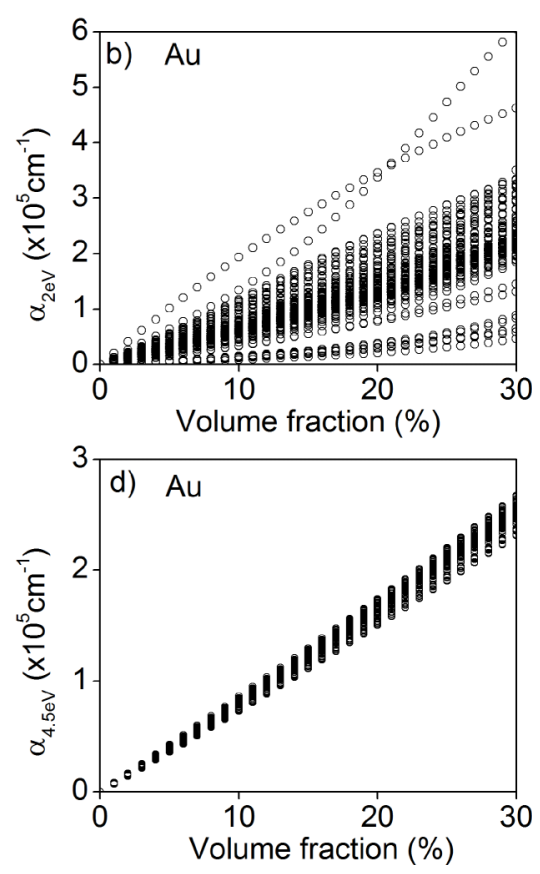

FIG. 5. Influence of the volume fraction on ((a) and (b)) $\alpha_{2} \mathrm{eV}$ and ((c) and

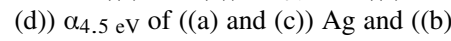
and (d)) Au NPs in water. The simulations are performed over 100 distributions. The standard deviation of the $\mathrm{L}_{\mathrm{i}}$ distribution varies in the $0-0.3$ range. 

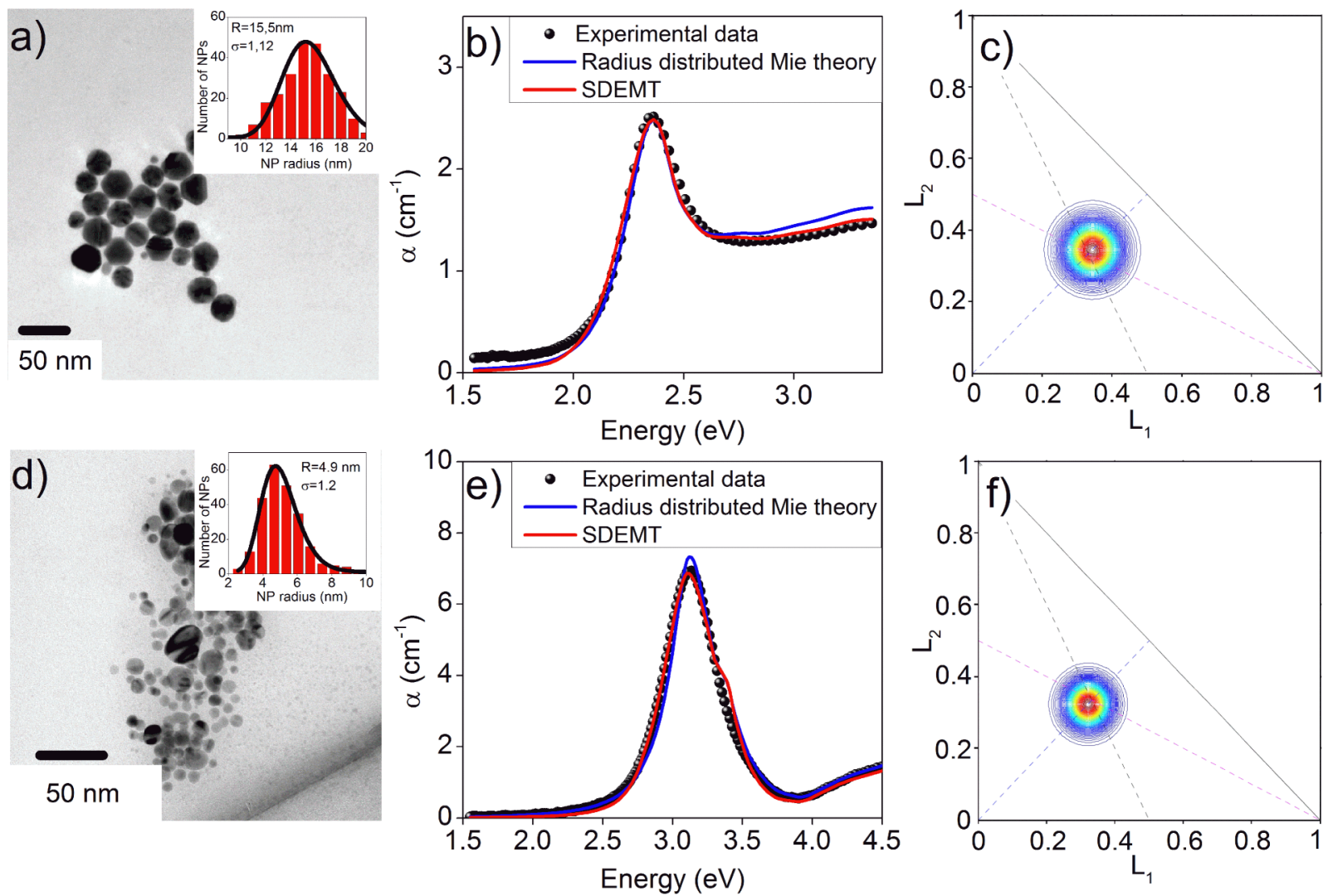

FIG. 6. ((a) and (d)) TEM images of (a) Au1 and (d) Ag1 NPs. In the inset, the NP radius distribution estimated from a statistical analysis of 250 NPs. ((b) and (e)) Comparison between the measured absorption spectra of (b) Au1 and (e) Ag1 NPs and the calculated ones from the radius distributed Mie theory and SDEMT. ((c) and (f)) Depolarization parameter distribution estimated from SDEMT of (c) Au1 and (f) Ag1.

$2.0 \times 10^{-4} \%$ and $2.4 \times 10^{-4} \%$, respectively. To improve the NP shape analysis, the experimental data are also adjusted with the SDEMT. The Levenberg-Marquardt algorithm is used to solve the inverse problem by minimizing the root mean square error between the measurements and the simulated data. First 6 free parameters are considered: the volume fraction, the mean values $\left(\bar{L}_{1} \bar{L}_{2}\right)$, and the standard deviations $\left(\sigma_{1}, \sigma_{2}, \sigma_{3}\right)$ of the distribution of depolarization parameters. Several parameters such as $\bar{L}_{1}$ and $\bar{L}_{2}$ or $\sigma_{1}$ and $\sigma_{2}$ are found to be close together. By calculating the correlation matrix between each free parameter, the number of free parameters is then iteratively reduced in order to avoid the correlation between them. By using this procedure, we finally find that $\bar{L}_{1}=\bar{L}_{2}$ and $\sigma_{1}=\sigma_{2}$. In other words, the fit was performed by considering only 4 uncorrelated free parameters: the volume fraction, the mean value $\bar{L}_{1}$, and the standard deviations $\left(\sigma_{1}, \sigma_{3}\right)$ of the distribution of depolarization parameters. Although gold and silver NPs have different mean radii, the absorption spectra calculated from the SDEMT model are close to the measured ones. The volume fractions of $\mathrm{Ag} 1$ and Au1 obtained from SDEMT are 2.1 $\times 10^{-4} \%$ and $2.6 \times 10^{-4} \%$. These values are similar to the ones obtained from the radius distributed Mie theory. Moreover, the SDEMT model gives a better description of the optical properties of Au1 than the radius distributed Mie theory, especially beyond the interband transition threshold (Fig. 6(b)). The two dimensional distribution of $\left(L_{1}, L_{2}\right)$ (Figs. 6(c) and 6(f)) found from the SDEMT model has a standard deviation smaller than 0.04 and is centered at $(0.32,0.32)$ and $(0.34$,
0.34) for Ag1 and Au1, respectively, confirming the spherical shape of these NPs. This suggests that SDEMT model can be used to estimate the shape distribution of gold and silver NPs.

The TEM images of Au2 and Au3 are depicted in Figures 7(a) and 7(d). Au2 is composed of gold nanorods which can be assimilated to prolate NPs. A small amount of spherical NPs with a radius smaller than $25 \mathrm{~nm}$ is also observed in Au2. On the other hand, Au3 NPs have a more complex shape than Au2. Indeed, Au3 consists in a mixture of spherical NPs with a $4 \pm 4 \mathrm{~nm}$ mean radius and elongated irregular NPs, which comes from the coalescence of NPs during the laser ablation. ${ }^{36}$ In first approximation, NPs which have an elongated irregular shape are considered as prolate NPs. The distributions of minor and major axes, i.e., the Ferret diameters of Au2 nanorods and Au3 elongated irregular NPs are extracted from a statistical analysis of TEM images performed over more than 100 particles. The depolarization parameter $\mathrm{L}_{\mathrm{i}}$ of $\mathrm{Au} 2$ nanoroad and Au3 elongated NPs can be calculated from the following equation by considering that $\mathrm{a}_{1}>\mathrm{a}_{2}=\mathrm{a}_{3}$ :

$$
L_{i}=\frac{a_{1} a_{2} a_{3}}{2} \int_{0}^{+\infty} \frac{d q}{\left(a_{i}^{2}+q\right) \sqrt{\left(a_{1}^{2}+q\right)\left(a_{2}^{2}+q\right)\left(a_{3}^{2}+q\right)}},
$$

where $a_{i}(i=1,2,3)$ are the lengths of the principal axes of the ellipsoids measured by TEM. The mean values of minor and major axes of Au2 nanorods obtained from TEM are $28 \pm 6 \mathrm{~nm}$ 

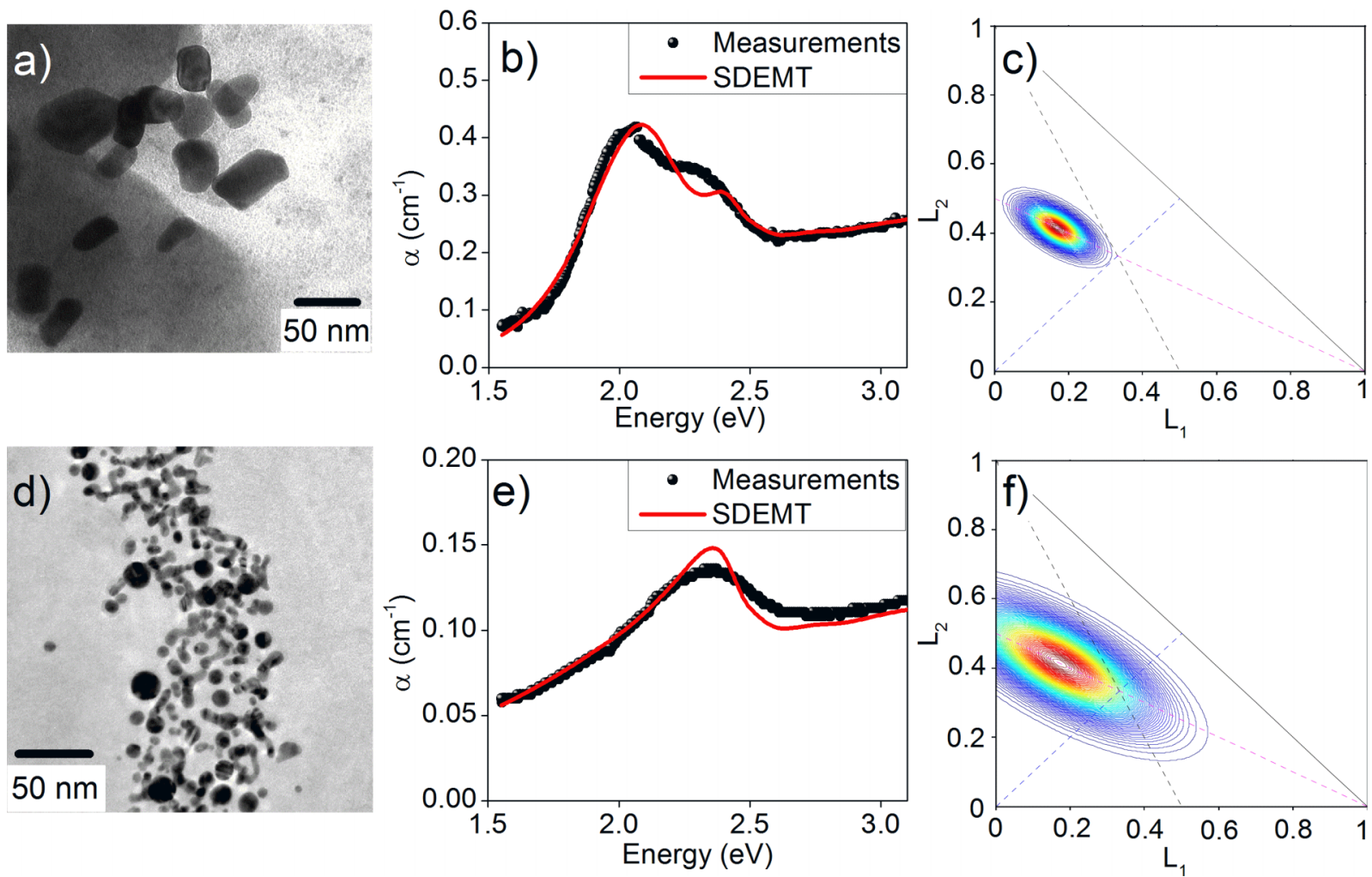

FIG. 7. ((a) and (d)) TEM images of (a) Au2 and (d) Au3 NPs. ((b) and (e)) Comparison between the measured absorption spectra of (b) Au2 and (e) Au3 NPs and the calculated ones from SDEMT. ((c) and (f)) Depolarization parameter distribution estimated from SDEMT of (c) Au2 and (f) Au3.

and $47 \pm 7 \mathrm{~nm}$, respectively. According to Eq. (16), the mean depolarization parameter is 0.4 .

The distribution of $\mathrm{L}_{1}$ depolarization parameter of gold nanorods follows a nearly Gaussian distribution with a 0.21 mean value (Figs. 8(a) and 8(b)). Au2 has a wider $\mathrm{L}_{1}$ distribution than Au3. Indeed, as shown on TEM image (Fig. 7(d)), Au3 NPs are highly distributed in shape. Note that the peak at 0.33 is attributed to spherical NPs. The Au2 NPs' spectrum (Fig. 7(b)) is characterized by two overlapped broad bands centered at $2.05 \mathrm{eV}$ and $2.32 \mathrm{eV}$. By considering Fröhlich condition (15), these bands are assigned to 0.15 and 0.3 depolarization parameters. In other words, the band centered at $2.32 \mathrm{eV}$ is the optical signature of spherical NPs, while the band centered at $2.05 \mathrm{eV}$ can be assimilated to the L-SPR band of $\mathrm{Au}$ nanorods. The absorption spectrum of Au3 (Fig. 7(e)) is characterized by a SPR band centered at $2.34 \mathrm{eV}$ which has a broad absorption tail in the visible spectral range. As discussed in Sec. III, this behavior traduces that Au3 NPs are highly distributed in shape.
Both spectra are fitted with the SDEMT model by using the same procedure as described for spherical NPs. The spectra calculated from the SDEMT model is close to the measured one. Some improvements of the Au2 fit, beyond the scope of this paper, can be obtained by using a bimodal distribution in order to take into account both nanorods and spherical NPs. In the visible range, the calculated absorption spectrum of Au2 has two bands centered at $2.07 \mathrm{eV}$ and $2.39 \mathrm{eV}$, attributed to the longitudinal and transversal plasmon resonances of gold nanorods. Due to the presence of spherical NPs and its small amplitude, the T-SPR band of Au2 nanorods is not clearly observed in the measured spectrum.

The NP distributions, obtained from the SDEMT model, are reported in Figures 7(c) and 7(f). The distributions are centered on the prolate axis $\left(\mathrm{L}_{1}=1-2 \mathrm{~L}_{2}\right)$ confirming that Au2 and $\mathrm{Au} 3$ are mainly composed of nanorods and elongated NPs which can be assimilated to prolate NPs. Moreover, the Au2 distribution is actually wider. Indeed, contrary to Au2, Au3 contains irregular elongated shapes which have different
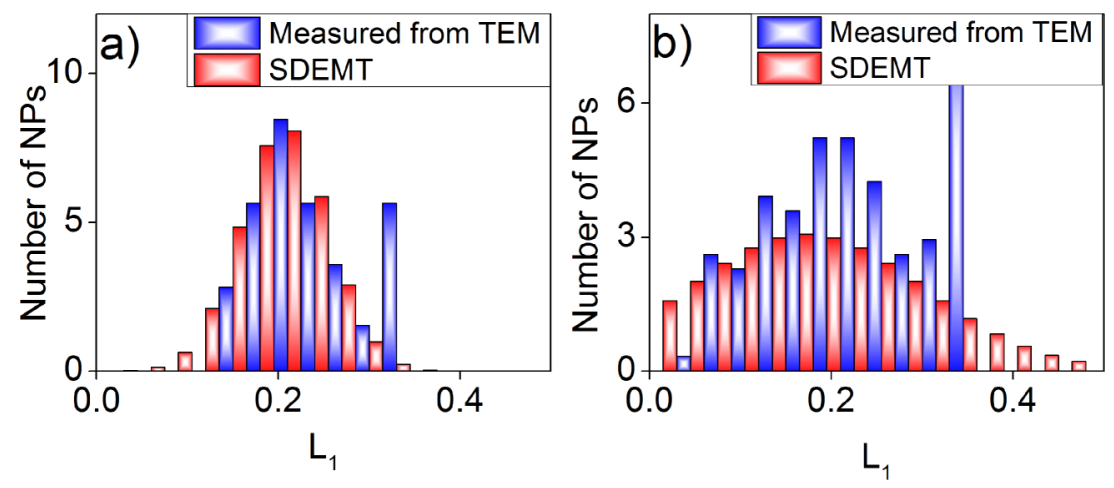

FIG. 8. Comparison between the (a) Au2 and (b) Au3 distributions of $\mathrm{L}_{1}$ depolarization parameter along the prolate axis estimated from SDEMT and the calculated ones from TEM images. 
depolarization parameters. The distributions of $\mathrm{L}_{1}$ along the prolate axis are compared with the estimated ones from TEM measurements (Fig. 8). To facilitate the comparison, the distribution obtained from the fit is normalized to the area under the distribution determined from TEM. The distribution along the prolate axis is similar to the ones extracted from the TEM images confirming the correctness of the SDEMT model. Moreover, this suggests that despite the complex form of Au3 NPs, the latter may be considered as spheroids. Thus, the SDEMT model can be used to extract some relevant information of the NPs' shape distribution. Contrary to TEM measurements, this characterization procedure is based on non-local and noncontact measurements and allows performing fast statistical analysis.

\section{CONCLUSIONS}

In summary, an effective medium theory based on a mean field and quasistatic approach is introduced to describe the optical properties of spheroids distributed in shape. This model was used to explain the impact of the shape distribution on the plasmon band of silver and gold NPs. The plasmon band position and width mainly depend on the mean value and standard deviation of depolarization parameters, respectively. In the interband spectral range, the NPs' volume fraction can be accurately estimated from the imaginary part of the effective dielectric function. The distribution of depolarization parameters is determined from the absorption spectra by fitting it with the SDEMT model. These distributions are close to the ones obtained from TEM measurements. Contrary to TEM measurements, this characterization procedure is compatible with in situ measurements. Thus, SDEMT model can give precious information about the NP shape distribution of colloidal solution.

${ }^{1}$ U. Kreibig and M. Vollmer, Optical Properties of Metal Clusters (Springer, Berlin, 1995)

${ }^{2}$ C. Noguez, J. Phys. Chem. C 111, 3806 (2007).

${ }^{3}$ Y. Li, K. Zhao, H. Sobhani, K. Bao, and P. Nordlander, J. Phys. Chem. Lett. 4, 1352 (2013).

${ }^{4}$ Z. Niu, Y.-R. Zhen, M. Gong, Q. Peng, P. Nordlander, and Y. Li, Chem. Sci. 2, 2392 (2011).

${ }^{5}$ A. D. McFarland and R. P. Van Duyne, Nano Lett. 3, 1057 (2003).
${ }^{6}$ R. Kitsomboonloha, C. Ngambenjawong, W. S. Mohammed, M. B. Chaudhari, G. L. Hornyak, and J. Dutta, Micro Nano Lett. 6, 342 (2011).

${ }^{7}$ K. R. Catchpole and A. Polman, "Plasmonic solar cells," Opt. Express 16, 21793 (2008).

${ }^{8}$ C. F. Bohren and D. R. Huffman, "Absorption and scattering by a sphere," in Absorption and Scattering of Light by Small Particles (Wiley, Germany, 1998).

${ }^{9}$ V. Amendola and M. Meneghetti, J. Phys. Chem. C 113, 4277 (2009).

${ }^{10}$ F. Bourquard, A. S. Loir, C. Donnet, and F. Garrelie, Appl. Phys. Lett. 104, 104101 (2014).

${ }^{11}$ S. Eustis and M. A. El-Sayed, J. Appl. Phys. 100, 044324 (2006).

${ }^{12}$ J. M. Garnett, Philos. Trans. R. Soc., A 205, 237 (1906).

${ }^{13}$ Y. Battie, N. Destouches, F. Chassagneux, D. Jamon, L. Bois, N. Moncoffre, and N. Toulhoat, Opt. Mater. Express 1, 1019 (2011).

${ }^{14}$ A. En Naciri, P. Miska, A. S. Keita, Y. Battie, H. Rinnert, and M. Vergnat, J. Nanopart. Res. 15, 1 (2013).

${ }^{15}$ J. Sukmanowski, Y. Battie, F. X. Royer, and A. En Naciri, J. Appl. Phys. 112, 103536 (2012)

${ }^{16} \mathrm{M}$. Losurdo and K. Hingerl, Ellipsometry at the Nanoscale (Springer, Berlin, 2013).

${ }^{17}$ Y. Battie, A. Resano-Garcia, N. Chaoui, Y. Zhang, and A. En Naciri, J. Chem. Phys. 140, 044705 (2014).

${ }^{18}$ A.-S. Keita, A. En Naciri, Y. Battie, F. Delachat, M. Carrada, G. Ferblantier, and A. Slaoui, J. Appl. Phys. 116, 103520 (2014).

${ }^{19}$ R. Sayah, L. Nadar, F. Vocanson, Y. Battie, S. Reynaud, R. Vera, A. Boukenter, and N. Destouches, Microporous Mesoporous Mater. 139, 45 (2011).

${ }^{20}$ Y. Battie, A. En Naciri, W. Chamorro, and D. Horwat, J. Phys. Chem. C 118, 4899 (2014).

${ }^{21}$ J. Toudert, L. Simonot, S. Camelio, and D. Babonneau, Phys. Rev. B 86, 045415 (2012).

${ }^{22}$ S. K. Mandal, R. K. Roy, and A. K. Pal, J. Phys. D: Appl. Phys. 36, 261 (2003).

${ }^{23}$ A. L. González, C. Noguez, and A. S. Barnard, J. Mater. Chem. C 1, 3150 (2013).

${ }^{24}$ A. L. González, C. Noguez, and A. S. Barnard, J. Phys. Chem. C 116, 14170 (2012).

${ }^{25}$ A. L. González, C. Noguez, J. Beránek, and A. S. Barnard, J. Phys. Chem. C 118, 9128 (2014).

${ }^{26}$ T. Yamaguchi, S. Yoshida, and A. Kinbara, Thin Solid Films 21, 173 (1974).

${ }^{27}$ A. V. Goncharenko, V. Z. Lozovski, and E. F. Venger, J. Phys.: Condens. Matter 13, 8217 (2001).

${ }^{28}$ A. V. Goncharenko and A. O. Pinchuk, Opt. Mater. Express 4, 1276 (2014).

${ }^{29}$ L. Gao, J. T. K. Wan, K. W. Yu, and Z. Y. Li, J. Phys.: Condens. Matter 12, $6825(2000)$.

${ }^{30}$ A. V. Goncharenko and E. F. Venger, Phys. Rev. E 70, 057102 (2004).

${ }^{31}$ E. D. Palik, Handbook of Optical Constants of Solids (Academic Press, Boston, 1998).

${ }^{32}$ P. B. Johnson and R. W. Christy, Phys. Rev. B 6, 4370 (1972).

${ }^{33}$ P. Winsemius, F. F. Van Kampen, H. P. Lengkeek, and C. G. Van Went, J. Phys. F: Met. Phys. 6, 1583 (1976).

${ }^{34}$ G. Leveque, C. G. Olson, and D. W. Lynch, Phys. Rev. B 27, 4654 (1983).

${ }^{35}$ B. Nikoobakht and M. A. El-Sayed, Chem. Mater. 15, 1957 (2003).

${ }^{36}$ S. E. Black, Laser Ablation: Effects and Applications (Nova Science Publishers, Inc., New York, 2011). 